\title{
Complete endoscopic radical resection of thyroid cancer via an oral vestibule approach
}

\author{
CONG BIAN, HUI LIU, XI-YU YAO, SHU-PING WU, YU WU, CHANG LIU and TIAN-SHUN HUANG \\ Department of Head and Neck Surgery of Fujian Cancer Hospital and Fujian Medical University Cancer Hospital, \\ Fuzhou, Fujian 350014, P.R. China
}

Received February 9, 2017; Accepted April 17, 2018

DOI: $10.3892 / \mathrm{ol} .2018 .9369$

\begin{abstract}
The aim of the present study was to explore the feasibility, safety and effectiveness of complete endoscopic radical resection of thyroid cancer via an oral vestibule approach. A total of 60 patients with unilateral thyroid papillary carcinoma were divided into two groups. Half of them underwent complete endoscopic surgeries via an oral vestibule approach at the Department of Head and Neck Surgery of Fujian Cancer Hospital between November 2014 and December 2016. The other 30 patients underwent traditional open surgeries. All the patients underwent unilateral lobectomy and central neck dissection. Tumor diameter, surgery duration, intraoperative inflation pressure and end-tidal $\mathrm{CO}_{2}$ flow rate, intraoperative peak value of the partial pressure of end-tidal $\mathrm{CO}_{2}$, postoperative extubation time, the number of lymph nodes in the specimens of central neck dissection and postoperative complications were noted. From this data, tumor diameter ( $\mathrm{T}$ stage of tumor), surgery duration, postoperative extubation time, the number of lymph nodes in the specimens of central neck dissection and postoperative complications were compared between the two groups. In the endoscopic group, 1 patient had a tracheal injury, and 1 patient had a submental skin perforation. Furthermore, 17 patients experienced transient numbness of the lower lip, 5 patients experienced an abnormal increase in the partial pressure of end-tidal $\mathrm{CO}_{2}$, and 2 patients experienced postoperative headache. No recurrent laryngeal nerve injury, postoperative bleeding, or infection was determined. There were no significant differences in all items of the indexes, compared with those patients who underwent open radical surgery. The lymph nodes from region VI may be well exposed and completely removed through this novel procedure with no visible scars, which not only ensured the surgery criterion was met, but also met the cosmetic requirements of the patients. The
\end{abstract}

Correspondence to: Dr Hui Liu, Department of Head and Neck Surgery of Fujian Cancer Hospital and Fujian Medical University Cancer Hospital, 420 Fuma Road, Fuzhou, Fujian 350014, P.R. China E-mail: happydoctor4huiliu@163.com

Key words: thyroid cancer, transoral vestibular approach, endoscopic thyroidectomy with central neck dissection, cosmetic, complication present study conducted procedures safely by surgeons highly skilled in performing laparoscopic surgery.

\section{Introduction}

The incidence of thyroid cancer has increased globally in the last 30 years (1). According to the data in 2015, its incidence is now the sixth highest among all female malignant tumor types in China (2). Generally, the prognosis of differentiated thyroid cancer is good, with the 15 -year survival rate being $>95 \%$ (2). With the improvement in survival rates, the demand for cosmetic salvage has increased, which has become a problem that cannot be ignored.

With the development of all types of endoscopic thyroid surgical procedures in which the incisions are not located in the neck, the scars in exposed areas of the body are prevented as much as possible. Currently, the method of surgery performed with a craniocaudal view has been adopted in the majority of types of endoscopic thyroid surgical procedures. However, the central lymph nodes cannot be sufficiently exposed and removed due to the shielding of the clavicle and sternum; therefore, this procedure does not meet the standard requirements of radical surgery for thyroid carcinoma (3-6). In order to avoid the bony shielding and allow the endoscopic radical surgery of thyroid cancer to comply with open surgery specifications, starting from November 2014 complete endoscopic thyroid surgery by an oral vestibule approach has been performed at the Fujian Cancer Hospital and the Fujian Medical University Cancer Hospital (Fuzhou, China). A total of 30 cases of papillary thyroid carcinoma (PTC) with stage I and II of the disease were included in the present study. Patients in the present study with thyroid cancer underwent a complete endoscopic radical resection by an oral vestibule approach. Patients ( $\mathrm{n}=30$ ), who underwent traditional open surgery, were compared with the aforementioned 30 cases of patients who underwent complete endoscopic surgery with an oral vestibule approach. The results of the present study indicated that except for the duration of the operation, there were no significant difference between the two groups.

\section{Patients and methods}

Patients. Between November 2014 and December 2016, among the patients with PTC treated at the Fujian Cancer 


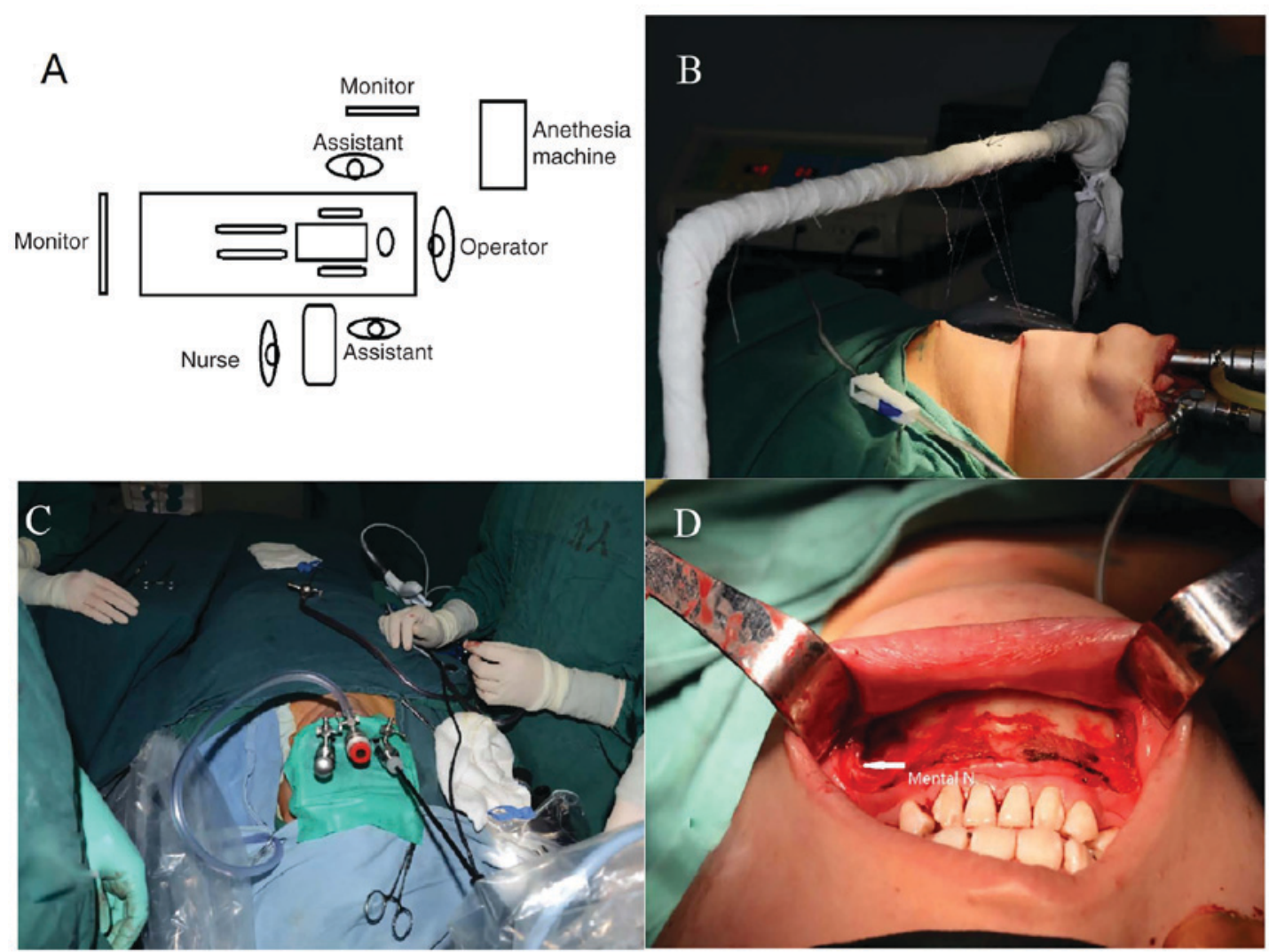

Figure 1. Procedures of the complete endoscopic radical surgery of thyroid cancer via an oral vestibule approach. (A) The operating room arrangement, depicting the operator stood over the head of patient and the monitor placed at the end of the table. (B) The suspended method was used to decrease the inflated pressure and flow to avoid hypercapnia. (C) Depicting the use of a rubber dam and the trocar arrangement. (D) The incision and mental nerve can be easily avoided, protecting the mental nerve.

Hospital and the Fujian Medical University Cancer Hospital, CT1/T2NOM0 and stage I/II (7) were included, and the cases with bilateral lobe lesions were excluded; at last 60 patients were selected. Written informed consent was obtained by all patients. Half of these patients underwent complete endoscopic thyroidectomy and central neck dissection via an oral vestibule approach; the other 30 patients underwent a traditional open surgery. From the endoscopic group, 1 patient was male and 29 patients were female; additionally, all the patients in the open group were females. The age of patients in the endoscopic group ranged between 17 and 41 years of age, with a median age of 24 years old; and in the open surgery group, the ages ranged between 17 and 44 years of age, with a median age of 26.5 years old. The tumor diameter in the endoscopic group was within $0.3-4.0 \mathrm{~cm}$, with a median diameter of $1.5 \mathrm{~cm}$; additionally, in the open group the tumor diameter was within $0.1-4.0 \mathrm{~cm}$, with a median diameter of $1.2 \mathrm{~cm}$. No clinical lymphatic and distant metastasis were observed in either group. Observational indexes included sex, age, pathological type, tumor size, duration time of the surgery, intraoperative bleeding volume, intraoperative inflation pressure and $\mathrm{CO}_{2}$ flow rate, intraoperative peak value of the partial pressure of end-tidal $\mathrm{CO}_{2}$, intraoperative tracheal injury, injuries of recurrent laryngeal nerve and mental nerve, skin injury, postoperative extubation time and postoperative infection. Furthermore, the duration of the surgery (based on the length of the surgery video), the bleeding volume, inflation pressure and $\mathrm{CO}_{2}$ flow rate and the peak value of the partial pressure of end-tidal $\mathrm{CO}_{2}$ during the surgery were obtained from anesthesia records.

Procedures of the complete endoscopic radical surgery of thyroid cancer via an oral vestibule approach

Posture. Patients were placed in the supine position, with a pillow under their shoulders and their neck hyper extended. The surgeon stood over the head of the patient.

Anesthesia. Patients were given intravenous and inhalant combined anesthesia through nasal intubation.

Incision design. A $1.5-\mathrm{cm}$ transverse incision was made to the gum on the lip side of the incisor teeth, which was adjacent to the turning point of the mucous membrane of the vestibular sulcus; subsequently, the mucoperiosteum was cut and separated along the labial surface of the bone to the inferior border of the mandible. An elevator was inserted through the incision, blunt separation was performed upon the superficial layer of deep cervical fascia to the bilateral sternoclavical joint, and two subcutaneous tunnels were formed and intersected at the hyoid plane. A $1.0-\mathrm{cm}$ trocar was inserted and inflated, and an observation lens was placed into it. Subsequently, it was inflated with $\mathrm{CO}_{2}$, with a pressure of $6-8 \mathrm{cmHg}$ and a flow rate of $10-12 \mathrm{l} / \mathrm{min}$. The other two incisions were produced near the turning point of the mucosa of the vestibular sulcus, which is located at the first premolar of each side. The separation layer and method were identical to that aforementioned and were 
Table I. Complications of endoscopic and open group.

\begin{tabular}{lcc}
\hline Complication & $\begin{array}{c}\text { Endoscopic } \\
(\mathrm{n}=30)\end{array}$ & $\begin{array}{c}\text { Open } \\
(\mathrm{n}=30)\end{array}$ \\
\hline Voice hoarse & 0 & 0 \\
Transient numbness of lower lip & 17 & - \\
Permanent numbness of lower lip & 0 & - \\
Skin perforation & 1 & - \\
Tracheal injury & 1 & 0 \\
Hypercapnia & 5 & - \\
Postoperative infection & 0 & 0 \\
Postoperative hemorrhage & 0 & 0 \\
\hline
\end{tabular}

-, no data; endoscopic, endoscopic surgery via vestibule group; open, traditional open surgery group.

intersected with the former tunnel. Subsequently, a $0.5-\mathrm{cm}$ trocar was placed through these two tunnels and used as the instrumental channel.

Space building. With the inflation of $\mathrm{CO}_{2}$, the flap was freed at the deep surface of the platysma, from the hyoid bone to the suprasternal notch and the bilateral sides reached the middle part of the sternocleidomastoid muscle. Following space building, the flap was suspended at the planes of the hyoid bone and cricoid cartilage with two No. 2-0 threads. Subsequently, the air pressure was decreased to $4 \mathrm{mmHg}$ to decrease the incidence of hypercapnia (Fig. 1).

Excision of the gland. The linea alba cervicalis was transected from the suprasternal notch to the hyoid plane, exposing the thyroid isthmus. Two special endoscopic thyroid retractors were implanted into the space via percutaneous puncture, and the strap muscles were retracted to the exterior margin of the thyroid. The trachea was exposed at the upper edge of thyroid isthmus, the vertebral lobe of the thyroid gland was dissected from the tracheal surface and the Delphian lymph node was excised. Blunt separation was performed between the trachea and thyroid isthmus, the isthmus was transected with ultrasonic scissors. The thyroid was dissected along the tracheal wall to the inner edge of the Berry's ligament. The suspensory ligament was cut off of the inner side of the thyroid gland. The upper pole of the gland was pulled to the inner upside and the surgical capsule of the thyroid was blunt dissected, exposing the vessels branch by branch. Then, the superior thyroid vessel was condensed segment by segment. Care was given in identifying and protecting the superior laryngeal nerve during this procedure. Following freeing the upper pole of the thyroid gland, the gland lobe was pulled to the inner upside and was blunt dissected and sharply separated closely to the thyroid gland membrane from upper pole to lower pole; then, the blood vessels were exposed and condensed branch by branch. The recurrent laryngeal nerve was dissected at the inferior point where the recurrent laryngeal nerve enters into the throat. The Berry's ligament and the gland pedicle were transected and the specimen was removed.
Central neck dissection. The lymphoid tissues in the central region was bluntly dissected at the surface of the trachea, and the fat lymphoid tissues in the central region was cut down at the internal edge of the cervical anterior muscle of the non-operative side down to the upper edge of the brachiocephalic artery. The fat lymphoid tissues on the surface of the common carotid artery were incised. The specimen was pulled to the inner upside, the recurrent laryngeal nerve was blunt dissected from cranium to caudal along the trend of the nerve and simultaneously, and the central fat lymphoid tissue was dissected along the esophageal surface up to the upper margin of the brachiocephalic artery. Subsequently, it was transversely cut at this plane and the specimens were removed.

The wound was washed, hemostasis was performed, the drainage tube was placed in the thyroid bed and was drawn off through the channel of the supraclavicular endoscopic thyroid retractor. The incision of the oral vestibule was sutured. The mental region and superior part of neck were pressure dressed with an elastic facemask.

Statistical analysis. The data were processed using SPSS (version 21.0; IBM Corp., Armonk, NY, USA). For non-normal distributions, Mann-Whitney $\mathrm{U}$ test and Wilcoxon $\mathrm{W}$ test was used. $\mathrm{P}<0.05$ was considered to indicate a statistically significant difference.

\section{Results}

In the present study, endoscopic cases were collected as an observation group, and traditional open surgery cases were collected as a control group. All data are presented in Tables I and II. There were no postoperative hemorrhage, infection or recurrent laryngeal nerve injury in the two groups. There was 1 case of submental skin perforation and 17 cases of transient numbness of the lower lip occurred in endoscopic group. All 17 patients with transient numbness of the lower lip recovered within 1-3 months. The partial pressure of end-tidal $\mathrm{CO}_{2}\left(\mathrm{PetCO}_{2}\right)$ exceeded $40 \mathrm{mmHg}$ in the first five cases during the endoscopic surgery; and the peak value of $\mathrm{PetCO}_{2}$ was $80 \mathrm{mmHg}$; however, the PetCO decreased to $40 \mathrm{mmHg}$ by decreasing the inflation pressure, increasing the oxygen flow, replacing the natrica calx and shutting off the pneumoperitoneum machine for 20-30 min. All the endoscopic surgeries were successfully completed; however, two cases of postoperative headache symptoms were determined and were rehabilitated following 1-2 days. No other serious complications were determined. Combined with flap suspension technology, the surgery was still successfully completed even when the inflation pressure was at $4 \mathrm{mmHg}, \mathrm{CO}_{2}$ flow rate was $6 \mathrm{l} / \mathrm{min}$ and $\mathrm{PetCO}_{2}$ pressure was decreased to $35-40 \mathrm{mmHg}$ during the entire process. When inflation pressure, inflation flow and duration of surgery were deduced, hypercapnia did not occur, except for the 15th patient. An accidental injury of the trachea was determined afterwards, and it was considered that the hypercapnia may have resulted from the re-absorption of $\mathrm{CO}_{2}$ leaking into the airway through the lungs. The surgery of the perforation site was successfully completed following the endoscopic suture and local spraying of biological fibrin glue for reinforcement. Postoperative recovery went well, the patient was extubated 
Table II. Compared endoscopic group with open group.

\begin{tabular}{|c|c|c|c|c|}
\hline Characteristic & Endoscopic $(\mathrm{n}=30)$ & Open $(n=30)$ & z-score & P-value \\
\hline Age, years & $17-41$ & $17-44$ & -0.496 & 0.620 \\
\hline Median & 24.00 & 26.5 & & \\
\hline Quartile range & 8.50 & 6.3 & & \\
\hline Tumor diameter, $\mathrm{cm}$ & $0.3-4.0$ & $0.1-4.0$ & -1.193 & 0.280 \\
\hline Median & 1.50 & 1.25 & & \\
\hline Quartile range & 0.50 & 0.73 & & \\
\hline T stage & & & 0.000 & 1.000 \\
\hline $\mathrm{T} 1$ & 23 & 23 & & \\
\hline $\mathrm{T} 2$ & 7 & 7 & & \\
\hline Blood loss, ml & $10-50$ & $10-50$ & -1.081 & 0.547 \\
\hline Median & 20.0 & 20.0 & & \\
\hline Quartile range & 5.0 & 10.0 & & \\
\hline Duration of surgery, min & $115-240$ & $37-90$ & -6.660 & 0.000 \\
\hline Median & 145.0 & 55.0 & & \\
\hline Quartile range & 28.8 & 10.0 & & \\
\hline Extubation time, days & $2-5$ & $3-6$ & -6.660 & 0.045 \\
\hline Median & 3 & 4 & & \\
\hline Quartile range & 1 & 1 & & \\
\hline Number of lymph nodes & $1-15$ & $0-13$ & -0.603 & 0.547 \\
\hline Median & 5 & 4.5 & & \\
\hline Quartile range & 1 & 2.5 & & \\
\hline
\end{tabular}

T stage used Mann-Whitney U test. Other results used Wilcoxon W test. Endoscopic, endoscopic surgery via vestibule group; open, traditional open surgery group.

and discharged on the 4th day, and no subcutaneous emphysema or infection occurred. Compared with the 30 patients who were undergoing a traditional open surgery, there was no significance in the age, the diameter of tumor and the proportion of patients with $\mathrm{T}$ stage between the two groups. The duration of endoscopic surgery was significantly longer compared with the open surgery $(\mathrm{P}=0.000)$. There was also a significant difference in extubation time $(\mathrm{P}=0.045)$, but the difference between the two groups was close. Furthermore, there was no significant difference in the bleeding volumes and the number of lymph nodes which were dissected in the central region (Table II). A drainage tube was regularly used following thyroid surgery. When the drainage was $<20 \mathrm{ml} /$ day, the drainage tube was extubated. Frequently, the extubating time was 4-5 days, and problems with vocal fold were never determined due to the long intubation periods.

\section{Discussion}

The surgical methods of radical surgery of thyroid cancer contained the traditional open surgery with Kocher's incision and the cosmetic surgery. Cosmetic surgery for thyroid tumor surgeries include ultrasound-guided ablation (8), minimally invasive video-assisted thyroidectomy $(9,10)$, endoscopic surgery with incision far from the neck (11-13) and endoscopic surgery via natural orifices $(12,13)$. In terms of cosmetics and minimally invasive effects, ultrasound-guided ablation has the most ideal qualities. However, the majority of thyroid surgeons oppose its application in the treatment of thyroid cancer. Minimally invasive incision surgery still results in scars in the neck and its cosmetic effect is poor (14). Complete endoscopic surgery may be conducted in a caudocranial direction or craniocaudal direction. The most common surgery direction for complete endoscopic thyroidectomy with incision out of neck was performed with a caudocranial view and there are two problems cannot be overcome when this was applied in the differentiated thyroid carcinoma: i) Scars remained on the chest wall, breast or axillary and ii) due to the shielding of the clavicle and sternum, the lymph nodes could not be sufficiently exposed in clearing the lymph nodes in the VI region (3).

In order to overcome the bony shielding, an approach with craniocaudal view is required, including a transoral approach and bidirectional approach of video-assisted neck surgery [BAVANSA and post auricular (PAA)]. Lee et al (15), Nakajo et al (5) and Wu et al (6) developed the BAVANSA. These procedures produced incisions in the chest wall, areola or axillary first for thyroidectomy and other incisions in PAA, precordial and submandibular and the floor of mouth (BAVANSA). All these have the ability to sufficiently expose, and therefore remove the lymph nodes of region VI, and even the lymph nodes from region II to IV. However, these procedures still produce a scar on the surface of body.

Endoscopic thyroid surgery via an oral approach is a natural orifice trans-luminal endoscopic surgery type, in 
which no scars are left on the body surface. Additionally, the shielding of the clavicle and sternum are avoided and lymph nodes in the VI region may be completely removed $(16,17)$. Endoscopic thyroidectomy via the approach of the floor of the mouth was proposed by Witzel et al (18), Benhidjeb et al (19), and Wilhelm and Metzig (20). This technique was introduced in China by Wu et al (21). Complete endoscopic thyroidectomy via an oral vestibular approach have been developed by Wang et al (22), Nitta et al (23), Nakajo et al (24) as well as others. Two surgical methods with and without gas inflation have been put forward.

Regarding the approach via the floor of the mouth, the endoscope may directly enter into the gap between the platysma muscle and strap muscle, and the anatomical layer is accurate. The interspace of three trocars is wider compared with using the oral vestibule approach, and the chopsticks effect does not easily occur (25). The limitation of this approach is that the endoscope must cross the triangle, which comprises of the jaw arch and hyoid, and contact with the three points of the trachea, hyoid bone and maxillary incisors. Therefore, movement in vertical directions is limited; and this approach cannot be applied in patients with limitations in extending their neck or opening their mouth (26). In addition, the lens is too close to the surgical site, which may cause smearing by atomized tissue fluid; therefore, this must be frequently cleaned. When the upper pole of the thyroid was treated, the instruments must go beyond the lens. These devices are interfered, mutually, which causes the exposure and treatment of the upper pole of the thyroid gland to be difficult. By the limited access of the mandible arch, the pathway for taking out the specimen cannot be expanded; therefore, the specimen must be split in order for it to be removed, even if it is only slightly larger (diameter $>3.0 \mathrm{~cm})(27,28)$, that the tumor cannot be removed en bloc violates the principle of being tumor-free. Finally, a $0.5-\mathrm{cm}$ endoscope was used for this approach, in which its brightness and visual field are not as good as that of the $1.0-\mathrm{cm}$ endoscope.

Similar to the oral vestibule approach, the endoscope is located at the lip-side of the mandible, the range of activities is wide (29), and the requirements of hyperextension of the neck and degree of mouth opening are lower. The endoscope is located above the plane of the instruments, with a large field of vision and less interference. This is beneficial to the exposure and treatment of the upper pole of the thyroid and Delphian lymph nodes. The incision may be extended along the two sides of the vestibular sulcus, which facilitates the complete removal of relatively large specimens (specimens $>4.0 \mathrm{~cm}$ can be completely removed). The brightness and visual field of the surgery area are good when the $1.0 \mathrm{~cm}$ endoscope is used. The disadvantages are as follows: Limited by the mouth width and mental nerve, the three trocars are relatively crowded and the chopsticks effect is significant; the vestibular sulcus mucus tore in the majority of cases; staying in the right plane of separation is difficult since the endoscope must pass two turning planes of the mental and hyoid. Therefore, the lower margin of the mandible should be beyond when the soft tissue was freed along the labial surface of the mandible. As a result, the obstruction of the mental may be avoided. Subsequently, the elevator was inserted at the lower edge of the mandible and was parallel to the skin of the mental triangle. The neck was required to be hyperextended when the elevator passed the hyoid plane, in order to align the sternoclavicular joint, throat and chin in the same plane, then the second obstruction in the plane of hyoid was avoided. The left hand was always placed in front of the neck to feel the dissection depth during the separation process. When the trocar was placed, rotation should be performed while pushing forward; the trocar should be properly placed by sliding into the correct position smoothly. Excessive force should not be used in order to avoid submental skin perforation.

As for the oral vestibular approach, leakage of $\mathrm{CO}_{2}$ may easily occur during the surgery; therefore, wet gauze may be filled around the trocar to decrease the leakage. Wang et al (29) moved the oral vestibule incision forward to the lingual side of the lower lip, which increased the interspace of the three trocars, and decreased the $\mathrm{CO}_{2}$ leakage, simultaneously.

The partial pressure of end-tidal $\mathrm{CO}_{2}$ indirectly reflects the changes of the partial pressure of $\mathrm{CO}_{2}$ in serum (30). Among the 30 patients in the present study, the partial pressure of end-tidal $\mathrm{CO}_{2}$ of 5/30 patients exceeded $40 \mathrm{mmHg}$ and postoperative headache symptoms appeared in $2 / 30$ cases. Accidental tracheal injury in 1 case was not detected promptly, and $\mathrm{CO}_{2}$ was re-absorbed by the airway, which resulted in the sharp increase of the partial pressure of end-tidal $\mathrm{CO}_{2}$ during the surgery. When the extreme datum in this case was excluded, it was determined that the trend of variation in the PetCO $\mathrm{O}_{2}$ during the surgery in the patients in the present study were consistent with the variation in the duration of surgery, the inflation flow rate and pressure of $\mathrm{CO}_{2}$ and the volumes of intraoperative bleeding. However, it appeared that there was no association with $\mathrm{CO}_{2}$ flow rate when it was $<111 / \mathrm{min}$. For the patients in the present study, when the surgery time was $<180 \mathrm{~min}$ and the inflation pressure of $\mathrm{CO}_{2}$ was $<6 \mathrm{mmHg}$, hypercapnia almost no longer occurred $(31,32)$. Intraoperative bleeding volume indirectly reflects intraoperative hemostasis. Increased bleeding volume indicated that intraoperative hemostasis was not performed thoroughly, which may lead to PetCO $\mathrm{O}_{2}$ increasing due to the $\mathrm{CO}_{2}$ directly entering into the blood. Therefore, in order to prevent hypercapnia, thorough hemostasis should occur during the surgery, inflation pressure and flow rate should be decreased $(<6 \mathrm{mmHg})$, and surgery time should be controlled within $180 \mathrm{~min}$. The space of endoscopic thyroidectomy via an oral approach was narrow, the discharge of smoke and fog was difficult and the visual field was unclear. Therefore, special exhaust passages should be set up for continuous low negative pressure suction in order to eliminate the smoke and save the waiting and cleaning times of the lens, improving operational efficiency (33). However, the surgical space was prone to collapse due to the decline of inflation pressure, the decrease in inflation flow rate and the continuous low-pressure vacuum suction of the exhaust passage. Based on the studies by Zhao et al (28) and Wang et al (29), inflation combined with suspension technologies were adopted, and the surgery space could be maintained if the skin was pulled up and suspended on the head rack following two needles of suture with No. 2-0 thread at the hyoid level and the cricoid cartilage level in the middle line of the neck; as a result, the surgery was successfully completed.

Transient numbness of the lower lip occurred in $17 / 30$ patients in the endoscopic group. It was considered 


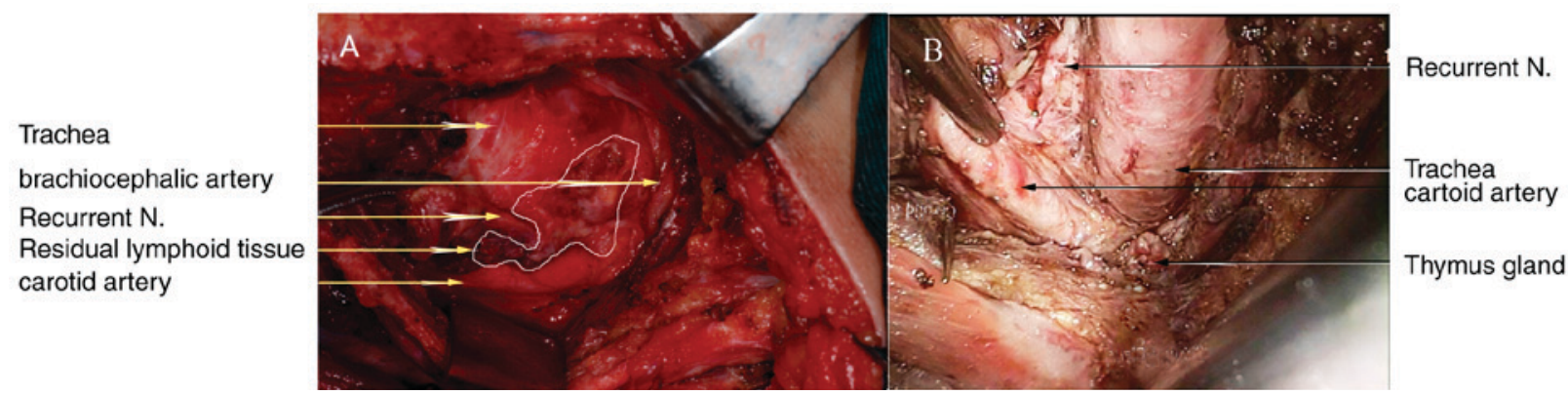

Figure 2. Endoscopic surgery. (A) Depicting the postoperative residual lymph node of endoscopic surgery via the chest wall and areola approach, this image was captured in a case of reoperation following a traditional open approach. (B) Post thyroidectomy and central neck dissection via endoscopic thyroid surgery with the transvestibular approach, this was similar to the surgery by an open approach.
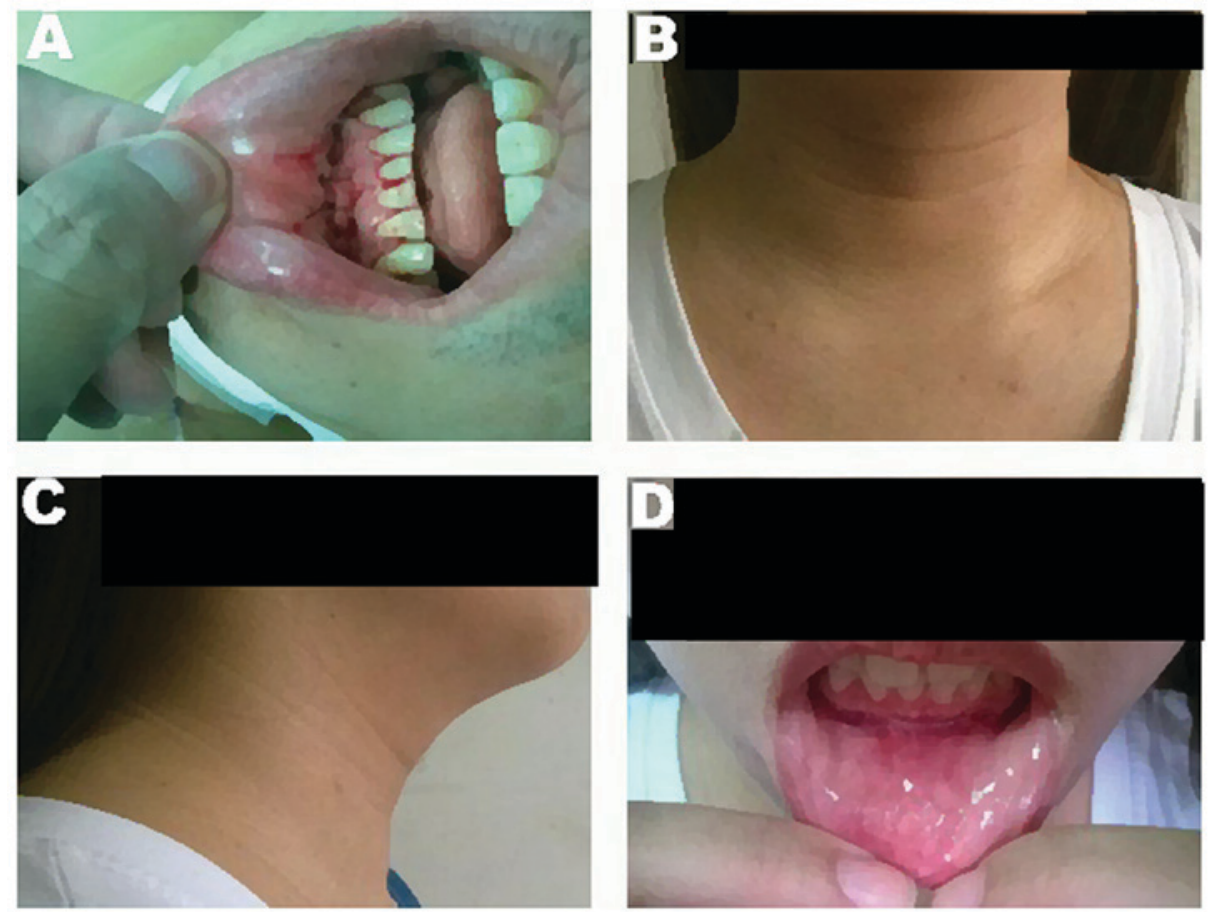

Figure 3. Postoperative view. (A) The suture in the vestibule at the time of completion of the surgery. (B) The frontal view a month later of the complete endoscopic surgery via the vestibule approach. (C) The side view a month later of the complete endoscopic surgery via the vestibule approach. There is no scar on the surface of body, depicting good cosmetic results. (D) The vestibular suture a month following the complete endoscopic surgery via the vestibule approach. The scar in the vestibule was almost invisible.

to be a result of the swing and traction of the nerve by the trocar, since it was visible as the trocar passed through the inner side of the mental nerve during the surgery. However, all the patients recovered in 1-3 months and there was no permanent numbness of the lower lip. It was determined in domestic and foreign autopsy studies that the mental foramen located in the buccal side of the first premolar had an average distance of $3.5 \mathrm{~cm}$ to the middle line. If the surgery channel on both sides moved into the labial side of canine teeth, within a $2.5 \mathrm{~cm}$ distance to the middle line, the probability of mental nerve injury may have been effectively decreased (34). In all 17 cases coupling with numbness of the lower lip, 12 of them occurred in the first 20 cases. When the mental nerve was dissected first, producing a $0.5-\mathrm{cm}$ instrument channel in the mental foramen in the later 15 cases, only $5 / 15$ experienced the transient number of lower lip. Thus, if it is possible to dissect the mental nerve first and produce a $0.5-\mathrm{cm}$ instrument channel at the labial side of canine, the incidences of transient numbness of lower lip may be significantly decreased

Compared with open surgery, there were no differences in surgery direction and method of dissection and protection of recurrent nerve and parathyroid glands, thyroidectomy and central neck dissection. Surgeons with skilled open surgical techniques and experience of endoscopic surgery are able to complete the radical surgery of thyroid cancer following the principles of open surgery without injuring the recurrent laryngeal nerve and parathyroid $(16,17)$.

Since the incision was altered into the mouth, and changed from class I to II, the risk of infection increased $(21,22,29)$. It is recommended that oral preparation should be performed, and antibiotics should be used to prevent infection during the perioperative period. The solutions were as follows: i) Perform supragingival scaling three days prior to the surgery; ii) gargle $15 \mathrm{ml}$ collutory of chlorhexidine and metronidazole compound, 
three times per day; iii) intravenous bolus of cefazolin sodium 1.030 min prior to anesthesia (an additional dose of the agent was added if surgery time was $>3 \mathrm{~h}$ ); iv) the oral cavity was insulated with a rubber barrier during the surgery; and v) a drainage tube was placed at the surgery area, and the mental region and superior part neck was dressed with an elastic face mask to prevent subcutaneous dropsy when the surgery was completed. There were no cases of infection from then on, which was consistent with the study by Rui et al (28).

The other complications of complete endoscopic radical surgery of thyroid cancer via an oral vestibular approach are primarily the same as endoscopic thyroidectomy via other approaches (28), including hypercapnia, subcutaneous emphysema, subcutaneous ecchymosis, incision infection, skin perforation, air embolism and others (35). Compared with other existing out of neck approaches of endoscopic thyroidectomy, the advantages of an oral vestibular approach of thyroid surgery are as follows: i) No scars were determined on body surface with good cosmetic effects (Fig. 2); and ii) there were no bony structural shielding, and the surgery range was consistent with that of open surgery (Fig. 3). The disadvantages are as follows: i) The surgery space was small and the instruments easy interfere with each other; ii) the smoke and fog in the surgery area is difficult to be eliminated, making the visual sight unclear and affecting the surgery schedule; iii) the treatment of the upper pole of the thyroid is relatively difficult; iv) energy and time is consumed; v) hypercapnia is likely to occur when the surgery is performed by beginners; vi) the probability of transient mental nerve injury is high; and vii) there is a risk of infection.

It was considered that the indications of complete endoscopic radical surgery of thyroid cancer via an oral vestibular approach are as follows: i) Patients who have a strong demand for cosmetic salvage and refuse any visible scars on the surface of body; ii) patients with cicatricial diathesis; iii) patients with differentiated thyroid cancer had below T2, thyroid volumes are approximately normal and no clinical cervical lymph node metastasis; and iv) for patients with benign thyroid tumors and the diameter of the tumor was $<4.0 \mathrm{~cm}$. It has been reported in literature that the thyroid tissue with the maximum volume of $>60 \mathrm{ml}$ may be incised by the approach via the floor of the mouth $(21,25,27)$. According to our experience, tumors with a diameter of $>4 \mathrm{~cm}$ may not be removed completely, and the risk of implantation is increased. Hyperthyroidism and Hashimoto's thyroiditis should be classified as relative contraindications for the relatively high difficulties and risks caused by the properties of crisp texture and easy bleeding of the tissues. The lymph nodes of regions VI, IV and the inferior part of III may be dissected by simple endoscopic surgery via an oral approach; however, the lymph nodes in region II and superior part of region III could not be touched (25). If the lymph nodes in region II and superior part of region III require treatment, approaches combined with chest wall or axillaries should be used (27).

The tracheal injury and submental skin perforation were the accident events that occurred in the present study. The authors have performed 100 cases of total endoscopic thyroid surgery and 60 cases via the vestibular approach. The tracheal injury and submental skin perforation have not occurred following those events. Having substantial experience and being careful enough means that the aforementioned complications may be avoided.
As a novel surgical procedure, the experiences of all the surgeons are less, and the security and standardization of radical surgery of thyroid cancer via an oral vestibule may be guaranteed as long as the indications were strictly followed $(4,36,37)$. However, the surgery is difficult, time-consuming, and requires rich open surgery experiences and skilled techniques of the endoscope (22). Therefore, it should not be promoted as a routine surgery procedure. However, it may be provided as a special surgery and service for the populations who have special cosmetic demands. This may be performed by a skilled specialist of thyroid surgery and endoscopic surgery at the thyroid center or the endoscopic surgery center. Further clinical studies are required for the expansion of the indications.

\section{Acknowledgements}

Not applicable.

\section{Funding}

The present study was supported by The National Clinical Key Specialty Construction Program.

\section{Availability of data and materials}

The datasets used and analyzed during the present study are available from the corresponding author on reasonable request.

\section{Authors' contributions}

CB and HL performed all of the endoscopic and open operations and were major contributors in writing the manuscript. XYY, SPW, YW, CL and TSH assisted in the operations and analyzed and interpreted the patient data. All authors read and approved the final manuscript.

\section{Ethics approval and consent to participate}

The study was approved by the Ethics Committee of Fujian Cancer Hospital (no. KT2017-013-01) and all patients provided their written informed consent.

\section{Patient consent for publication}

Written informed consent has been obtained from all patients, declaring that any associated data and accompanying images can be published. Personally identifiable information will be treated confidentially.

\section{Competing interests}

There is no competing interests in the present study.

\section{References}

1. Pellegriti G, Frasca F, Regalbuto C, Squatrito S and Vigneri R: Worldwide increasing incidence of thyroid cancer: Update on epidemiology and risk factors. J Cancer Epidemiol 2013: 965212, 2013. 
2. Chen W, Zheng R, Baade PD, Zhang S, Zeng H, Bray F, Jemal A Yu XQ and He J: Cancer statistics in china, 2015. CA Cancer J Clin 66: 115-132, 2016.

3. Wang P: Present situation and prospect of endoscopic radical surgery of thyroid cancer. J Surg Concepts and Practice: 522-525, 2011 (In Chinese)

4. Cho MJ, Park KS, Cho MJ, Yoo YB and Yang JH: A comparative analysis of endoscopic thyroidectomy versus conventional thyroidectomy in clinically lymph node negative thyroid cancer. Ann Surg Treat Res 88: 69-76, 2015.

5. Nakajo A,Arima H,Hirata M, Yamashita Y, Shinden Y,HayashiN, Kawasaki Y, Arigami T, Uchikado Y, Mori S, et al: Bidirectional approach of video-assisted neck surgery (BAVANS): Endoscopic complete central node dissection with craniocaudal view for treatment of thyroid cancer. Asian J Endosc Surg 10: 40-46, 2017

6. Wu GY, Fu JB, Yan W, Luo ZZ, Lin FS, Kang PZ, Hong XQ, Chen QG and Lin E: Endoscopic central neck dissection of thyroid cancer with transbrest and tansoral combined approach. China J Surg, 54: 297-298, 2016

7. Liu SS, Wei B, Yang WJ and Step M: Introduction to the American Cancer Federation Breast Cancer Staging (Seventh Edition). Chinese J Pathol 11: 787-790, 2010 (In Chinese).

8. Jeong WK, Baek JH, Rhim H, Kim YS, Kwak MS, Jeong HJ and Lee D: Radiofrequency ablation of benign thyroid nodules: Safety and imaging follow-up in 236 patients. Eur Radiol 18: 1244-1250, 2008

9. Ding Z, Deng X, Fan Y and Wu B: Single-port endoscopic thyroidectomy via a submental approach: Report of an initial experience. Head Neck 36: E60-E64, 2014.

10. Radford PD, Ferguson MS, Magill JC, Karthikesalingham AP and Alusi G: Meta-analysis of minimally invasive video-assisted thyroidectomy. Laryngoscope 121: 1675-1681, 2011.

11. Lee DY, Baek SK and Jung KY: Solo-surgeon retroauricular approach endoscopic thyroidectomy. J Laparoendosc Adv Surg Tech A 27: 63-66, 2017

12. Pai VM, Muthukumar P, Prathap A, Leo J and A R: Transoral endoscopic thyroidectomy: A case report. International J Surg Case Rep 12: 99-101, 2015.

13. Wong KP and Lang BHH: Endoscopic thyroidectomy: A literature review and update. Curr Surg Rep 1: 7-15, 2013.

14. Wang P and Yan H: Application of laparoscopic surgery in differentiated thyroid cancer surgery. Med \& Philosophy 20: 14-16, 2012 (In Chinese)

15. Lee KE, Kim HY, Park WS, Choe JH, Kwon MR, Oh SK and Youn YK: Postauricular and axillary approach endoscopic neck surgery: A new technique. World J Surg 33: 767-772, 2009.

16. Park JO, Kim CS, Song JN, Kim JE, Nam IC, Lee SY, Chun BJ, Cho JH, Joo YH, Cho KJ, et al: Transoral endoscopic thyroidectomy via the tri-vestibular routes: Results of a preclinical cadaver feasibility study. Eur Arch Otorhinolaryngol 271: 3269-3275, 2014

17. Guo P, Tang Z, Ding Z, Chu G, Yao H, Pan T and Wang H: Transoral endoscopic thyroidectomy with central neck dissection: Experimental studies on human cadavers. Chin Med J (Engl) 127: 1067-1070, 2014

18. Witzel K, von Rahden BH, Kaminski C and Stein HJ: Transoral access for endoscopic thyroid resection. Surg Endosc 22: 1871-1875, 2008.

19. Benhidjeb T, Wilhelm T, Harlaar J, Kleinrensink GJ, Schneider TA and Stark M: Natural orifice surgery on thyroid gland: Totally transoral video-assisted thyroidectomy (tovat) Report of first experimental results of a new surgical method. Surg Endosc 23: 1119-1120, 2009.

20. Wilhelm T and Metzig A: Video. Endoscopic minimal-invasive thyroidectomy: First clinical experience. surg endosc. Surg Endosc 24: 1757-1758, 2011.
21. Wu GY, Fu J, Luo Z, Chen Q, Lin E, Hong X, Lin F and Yan W: Totally transoral video-assisted thyroidectomy: report of 37 cases. Chin J General Surg: 32-34, 2014.

22. Wang C, Zhai H, Liu W, Li J, Yang J, Hu Y, Huang J, Yang W, Pan Y and Ding H: Thyroidectomy: A novel endoscopic oral vestibular approach. Surgery 155: 33-38, 2014.

23. Nitta K, Ishikawa N, Kawaguchi M, Ooi A and Watanabe G: Thyroidectomy using pure natural orifice transluminal endoscopic surgery in a porcine model. Artif Organs 39: 441-445, 2015.

24. Nakajo A, Arima H, Hirata M, Mizoguchi T, Kijima Y, Mori S, Ishigami S, Ueon S, Yoshinaka $\mathrm{H}$ and Natsugoe S: Trans-oral video-assisted neck surgery (TOVANS). A new transoral technique of endoscopic thyroidectomy with gasless premandible approach. Surg Endosc 27: 1105-1110, 2013.

25. Su YH, Tang ZP, Ding ZH, Wang HQ, Wu T, Liang JS, Cen H, Zhao XW, Ding JW, Hua W and Tang C: Total trans-oral endoscopic thyroidectomy and cervical lymphadenectomy: A human cadavers surgery study. Zhonghua Wai Ke Za Zhi 51: 552-555, 2013 (In Chinese).

26. Jing-Hong X: Application progress of totally trans-oral video-assisted thyroidectomy. J Minimally Invasive Med, 11: 378-380, 2016.

27. Guo-Yang W and Jin-Bo Fu: The main technical points surgery of totally transoral video-assisted thyroidectomy. J Clin Surgery 23: 497-199, 2015.

28. Zhao L, Li JY and Wang CC: The research progress of endoscopic thyroidectomy through the mouth. Chinese Journal of Surgery 52: 541-543, 2014.

29. Wang C, Di H, Liu W, Li J, Yang J, Hu Y, Cai N, Yang Hua: Transoral-vestibule endoscopic thyroidectomy: Experience in 6 cases. China J Endoscopy: 363-366, 2013 (In Chinese).

30. Liang KH, Zheng HL, Zhang J and Ma BX: Anesthesia management of Totally transoral video-assisted thyroidectomy. Hainan Medical Journal, 26: 2924-2925, 2015.

31. Ochiai R, Takeda J, Noguchi J, Ohgami M and Ishii S: Subcutaneous carbon dioxide insufflation does not cause hypercarbia during endoscopic thyroidectomy. Anesth Analg 90: 760-762, 2000.

32. Kim JA, Kim JS, Chang MS, Yoo YK and Kim DK: Influence of carbon dioxide insufflation of the neck on intraocular pressure during robot-assisted endoscopic thyroidectomy: A comparison with open thyroidectomy. Surg Endosc 27: 1587-1593, 2013

33. Li JY, Wang CC, Pan YL, Cheng J, Hu YZ, Yang JG, Huang J and Shen YY: Complete endoscopic thyroidectomy: introduction and clinical application of smog management during surgery. Journal of Jinan University (Natural Science and Medicine Edition), 33: 180-182, 2012.

34. Huang YX, Cai CZ, Zhang T, Chai L, Wang G, Chang T, Shi LX and Fang L; Department of General Surgery, Tenth People's Hospital of Tongji University: Anatomical study of transoral thyroidectomy. Chin J Clin Anatomy: 153-155, 2014.

35. Rajan S, Paul J and Kumar L: Carbon dioxide embolism during endoscopic thyroidectomy. Indian J Anaesth 60: 65-66, 2016.

36. Yang J, Wang C, Li J, Yang W, Cao G, Wong HM, Zhai H and Liu W: Complete endoscopic thyroidectomy via oral vestibular approach versus areola approach for treatment of thyroid diseases. J Laparoendosc Adv Surg Tech A 25: 470-476, 2015.

37. Wan WK, Kim JS, Hur SM, Kim SH, Lee SK, Choi JH, Kim S, Lee JE, Kim JH, Nam SJ, et al: Is robotic surgery superior to endoscopic and open surgeries in thyroid cancer? World J Surg 35: 779-784, 2011.

This work is licensed under a Creative Commons Attribution 4.0 International (CC BY 4.0) License. 